A note on polynomial diagonalization and Wiener-Hopf factorization of 2x2 matrices.

A. D. Rawlins 
A note on polynomial diagonalization and Wiener Hopf

$$
\text { factorization of } 2 \times 2 \text { matrices }
$$

by

A. D. Rawlins

Department of Mathematics and Statistics

Brunel University

Kingston Lane

Uxbridge

Middlesex UB8 3PH

Abstract

Given the matrix $G(\alpha)=\left[\begin{array}{ll}a(\alpha( & b(\alpha) \\ c(\alpha) & d(\alpha)\end{array}\right]$ it is shown that if

$b(\alpha) /\left\{\left(a(\alpha)-d(\alpha) \pm[a(\alpha)-d(\alpha))^{2}+4 b(\alpha) c(\alpha)\right]^{1}\right\}^{1}$ can be exp ressed

as a ratio of polynomials then the matrix G(a)can be explicitly WienerHopf factorized. The Wiener-Hopf factors will also have algebraic growth at infinity. 
1. Introduction

Recently there have appeared a number of papers dealing with the explicit Wiener-Hopf factorization of special types of matrices. Lebre[l]uses a rather elaborate procedure to factorize the matrix

$$
G_{1}(\alpha)=\left[\begin{array}{ccc}
1 & a p & 2 \\
a & 1
\end{array}\right]
$$

where $\mathrm{a}(\alpha)$ is an arbitrary function and $\mathrm{p}(\alpha)$ is a rational polynomial. Meister and speck[2][3] factorized the matrix

$$
\mathrm{G}_{2}(\alpha)=\beta_{1}(\alpha) \mathrm{R}_{1}(\alpha)+\beta_{2}(\alpha) \mathrm{R}_{2}(\alpha)
$$

where $\beta_{1}(\alpha)$ and $\beta_{2}(\alpha)$ are arbitrary scalar functions and $R_{1}(\alpha)$ and $R_{2}(\alpha)$ are matrices with rational elements such that

$$
\mathrm{R}_{1}^{2}=\mathrm{R}_{1}, \mathrm{R}_{2}=\mathrm{I}-\mathrm{R}_{1} .
$$

Meister and speck applied these results to the explicit factorization of the matrix

$$
G_{3}(\alpha)=\frac{1}{k_{2}^{2} t \frac{2}{2}}\left[\begin{array}{lcc}
\alpha{ }^{2} \psi-k_{2}^{2} t \frac{2}{2} & \alpha \xi_{2} \psi \\
\alpha \xi_{2} \psi & \xi_{2}^{2} \psi-k_{2}^{2} t \frac{2}{2}
\end{array}\right]
$$

Where $\psi=4 \tau-3 k_{2}^{2}, \tau=\alpha^{2}+\xi_{2}^{2}-t_{1} t_{2}$, which occurs in elastodynamic problems Kupradze[4]. Hurd and Luneburg[5]carried out a rather complicated factorization procedure for the matrix (which occurs in a problem of diffraction by an anisotropic impedance half plane)

$$
\mathrm{G}_{4}(\alpha)=\left[\begin{array}{cc}
1+\frac{\mathrm{k}^{2}-\alpha^{2}}{\mathrm{kn} \Gamma} & -\frac{\mathrm{k}_{2} \alpha}{\mathrm{kn \eta \Gamma}} \\
-\frac{\mathrm{k}_{3} \alpha}{\mathrm{kn}_{2} \Gamma} & 1+\frac{\mathrm{k}^{2}}{\mathrm{kn}_{2} \Gamma}
\end{array}\right]
$$

where $\Gamma=\left[\mathrm{k}^{2}-\alpha^{2}\right]_{\frac{1}{2}}^{1}, \mathrm{k}^{2}=\mathrm{k}^{2}-\mathrm{k}_{3}^{2}$. However, in a later paper Luneburg et al[6] they did realise that this matrix could be diagonalized by means 
Of polynomial matrices. In the paper* [6] they simply state the factorization of the matrix(which occurs in a diffraction problem by a partially coated dialectric slab):

$$
G 5(\alpha)=\left[\begin{array}{lc}
\frac{k_{1}^{2}-k_{z}^{2}}{\beta_{1}}+\frac{\mu}{\beta_{2}}\left(k_{2}^{2}-k_{z}^{2}\right) & \frac{\alpha}{\beta_{1}} k_{z}+\frac{\mu}{\beta_{2}} \alpha k_{z} \\
\frac{\alpha k_{z}}{\beta_{1}}+\frac{\mu}{\beta_{2}} \alpha k_{z} & \frac{k_{1}^{2}-\alpha^{2}}{\beta_{1}}+\frac{\mu}{\beta_{2}}\left(k_{2}^{2}-k_{z}^{2}\right)
\end{array}\right]
$$

where $\beta_{j}=\left(k_{j}^{2}-\alpha^{2}\right)^{\frac{1}{2}}, k_{j}=\left(k_{j}^{2}-k_{z}^{2}\right)^{\frac{1}{2}} \quad j=1,2$, in the form

$$
\begin{gathered}
\mathrm{G}_{5}(\alpha)=\mathrm{T}^{-1}(\alpha) \wedge(\alpha) \mathbf{T}(\alpha) \\
\mathrm{T}(\alpha)=\left[\begin{array}{cc}
\alpha & \mathrm{k}_{\mathrm{z}} \\
-\mathrm{k}_{2} & \alpha
\end{array}\right], \quad \Lambda(\alpha)=\left[\begin{array}{cc}
\frac{\mathrm{k}_{1}^{2}}{\beta_{1}}+\frac{\mu \mathrm{k}^{2}}{\beta_{2}} & 0 \\
0 & \beta_{1}+\mu \beta_{2}
\end{array}\right]
\end{gathered}
$$

The present paper shows that all the above matrices $G_{j}(\alpha), j=1,2,3,4,5$ fall into a more general class which can be Wiener-Hopf factorized by elementary means. We also give a simple criterion on the coefficients of the general matrix $G(\alpha)$ given by

$$
G(\alpha)=\left[\begin{array}{ll}
a(\alpha) & b(\alpha) \\
c(\alpha) & d(\alpha)
\end{array}\right]
$$

which will ensure that the matrix can be polynomially diagonalized. That is that $G(a)$ can be represented in the form

$$
G(\alpha)=P(\alpha) D(\alpha) P^{I}(\alpha)
$$

where $P(\alpha)$ and $P^{I}(\alpha)$ have only polynomial elements. $P^{I}(\alpha)=\operatorname{det}(P) P^{-}$

* They represent these factors as $\mathrm{G}_{-}(\alpha)=\mathrm{T}_{(\alpha)}^{-1} \Lambda_{-}(\alpha), \mathrm{G}_{+}(\alpha)=\Lambda_{+}(\alpha) \mathrm{T}(\alpha)$ Their factorization would seem to be not strictly correct because $\mathrm{T}^{-1}(\alpha)$ will have poles at $\mathrm{a}= \pm \mathrm{ik}$. However, in the application they eventually only require $G_{+} \alpha$ ) and $G_{-}^{-1}(\alpha)=\wedge(\alpha) \mathbf{T}(\alpha)$ to be analytic and regular in their regions of reguality, and hence their procedure gives the correct results. 
is the "formal inverse", and $D(\alpha)=\operatorname{diag}\left\{d,(\alpha), d_{2}(\alpha)\right\}$ where $d,(\alpha)$ and $d_{2}(\alpha)$ are arbitrary functions of $\alpha$. Obviously once such a decomposition as (1.10) has been achieved then the Wiener-Hopf factorization can be carried out quite simply as

$$
G(\alpha)=G_{+}(\alpha) G_{-}(\alpha)
$$

where

$$
G_{+}(\alpha)=P(\alpha) D_{+}(\alpha) \quad G_{-}(\alpha)=D_{-}(\alpha) P^{I}(\alpha)
$$

$\mathrm{D}_{ \pm}=\operatorname{diag}\left\{\mathrm{d}_{1} \pm(\alpha), \mathrm{d}_{2} \pm(\alpha)\right\}, \quad \mathrm{d}_{1}=\mathrm{d}_{1}+(\alpha) \mathrm{d}_{1}-(\alpha), \quad \mathrm{d}_{2}(\alpha)=\mathrm{d}_{2}+(\alpha) \mathrm{d}_{2}-(\alpha)$ We also observe that the factorization processes ensures that the factors will have algebraic growth at infinity, and thus enable complete solution of the relevant Wiener-Hopf problem to be carried out.

\section{Polynomial diagonalization}

Consider the matrix

$$
\mathrm{G}(\alpha)=\left[\begin{array}{ll}
a(\alpha) & b(\alpha) \\
c(\alpha) & d(\alpha)
\end{array}\right]
$$

The characteristic equation of this matrix is given by $\operatorname{det}(G-\lambda I)=0$, i.e. $\lambda^{2}-\lambda(a+d)+a d-b c=0$. Thus the characteristic values of $G$ are given by

$$
\lambda_{1}(\alpha)=\frac{(a+d)-\left\{(a-d)^{2}+4 b c\right\}^{\frac{1}{2}}}{2}, \quad \lambda_{2}(\alpha)=\frac{(a+d)-\left\{(a-d)^{2}+4 b c\right)^{\frac{1}{2}}}{2} .
$$

The corresponding eigenvetors are given by the solutions of the equations

$$
\begin{array}{ll}
\left(a-\lambda_{1}\right) v_{11}+b v_{21}=0, \quad c v_{11}+\left(d-\lambda_{1}\right) v_{21}=0 & \text { for } \lambda_{1} \\
\left(a-\lambda_{2}\right) v_{12}+b v_{22}=0, \quad c v_{12}+\left(d-\lambda_{2}\right) v_{22}=0 & \text { for } \lambda_{2} .
\end{array}
$$

Since the determinant of G-XI vanishes, the 2 equations in (2.3) are linearly dependent. Hence taking any one, say (2.3), we have

$$
\frac{\mathrm{v}_{11}(\alpha)}{\mathrm{v}_{21}(\alpha)}=\frac{-2 \mathrm{~b}}{(\mathrm{a}-\mathrm{d})-\left\{(\mathrm{a}-\mathrm{d})^{2}+4 \mathrm{bc}\right\}^{\frac{1}{2}}}
$$


Now, if we can diagonalise, the first column of the transformation matrix will consist of $\mathrm{V}_{11}(\alpha)$ and $\mathrm{V}_{12}(\alpha)$, and we require them to be polynomials. Thus if

$$
\frac{-2 b}{\left.(a-d)-\left\{(a-d)^{2}+4 b c\right\}\right)^{\frac{1}{2}}}=\frac{P_{11}(\alpha)}{P_{21}(\alpha)}
$$

where $\mathrm{P}_{11}(\alpha)$ and $\mathrm{P}_{21}(\alpha)$ are polynomials, then we can choose

$$
\mathrm{V}_{11}(\alpha)=\mathrm{P}_{11}(\alpha), \quad \mathrm{V}_{21}(\alpha)=\mathrm{P}_{21}(\alpha) \text {. }
$$

Similarly, solving either of the equations (2.4) we get for the components of the eigenvector corresponding to $\lambda_{2}$ that

$$
\frac{v_{12}}{v_{22}}=\frac{-2 b}{(a-d)+\left\{(a-d)^{2}+4 b c\right\}^{\frac{1}{2}}} ;
$$

and if

$$
\frac{-2 b}{(a-d)+\left\{(a-d)^{2}+4 b c\right\}^{\frac{1}{2}}}=\frac{P_{12}(\alpha)}{P_{22}(4 \alpha)},
$$

where $P_{12}(\alpha)$ and $P_{22}(\alpha)$ are polynomials then we can choose

$$
\mathrm{V}_{12}(\alpha)=\mathrm{P}_{12}(\alpha), \quad \mathrm{V}_{22}(\alpha)=\mathrm{P}_{22}(\alpha) .
$$

Thus if (2.6) and (2.8) (where these expressions must have distinct values in order to be able to diagonalize) are satisfied then $G(\alpha)$ can be represented by

$$
G(\alpha)=P(\alpha) D(\alpha) P^{I}(\alpha)
$$

where

$P I(\alpha)=\left[\begin{array}{c}P_{22}(\alpha)-P_{12}(\alpha) \\ -P_{21}(\alpha) P_{11}(\alpha)\end{array}\right], P(\alpha)=\left[\begin{array}{ll}P_{11}(\alpha) & P_{12}(\alpha) \\ P_{21}(\alpha) & P_{22}(\alpha)\end{array}\right], D(\alpha)=\left[\begin{array}{cc}\frac{\lambda_{1}(\alpha)}{\operatorname{det}(P)} & 0 \\ 0 & \frac{\lambda_{2}(\alpha)}{\operatorname{det}(P)}\end{array}\right]$,

and the Wiener-Hopf factorization can be carried out to give (1.11) and (1.12).

\section{Special cases of the general matrix}

(a) If we let $\mathrm{a}(\alpha)=1, \mathrm{~d}(\mathrm{a})=1, \mathrm{~b}(\alpha)=\mathrm{c}(\alpha) \mathrm{p}^{2}(\alpha)$ where $\mathrm{p}(\alpha)$ is a rational 
polynomial then we get the matrix considered by Lebre[1]. The expressions (2.5) and (2.7) now become

$$
\frac{\mathrm{v}_{11}(\alpha)}{\mathrm{v}_{21}(\alpha)}=\rho, \quad \text { and } \quad \frac{\mathrm{v}_{12}(\alpha)}{\mathrm{v}_{22}(\alpha)}=-\rho,
$$

so clearly we can polynomially diagonalize.

(b) If we let

$$
\begin{array}{ll}
a(\alpha)=\beta_{1}(\alpha) r_{11}(\alpha)+\beta_{2}(\alpha) \widetilde{r}_{11}(\alpha), \quad b(\alpha)=\beta_{1}(\alpha) r_{12}(\alpha)+\beta_{2}(\alpha) \widetilde{r}_{12}(\alpha) \\
c(\alpha)=\beta_{1}(\alpha) r_{21}(\alpha)+\beta_{2}(\alpha) \widetilde{r}_{21}(\alpha) & d(\alpha)=\beta_{1}(\alpha) r_{22}(\alpha)+\beta_{2}(\alpha) \widetilde{r}_{22}(\alpha)
\end{array}
$$

where $r_{i j}(\alpha)$ and $\widetilde{r}_{i j}(\alpha)$ are the rational elements of the matrices $R_{1}$ and $\mathrm{R}_{2}$ respectively, then we obtain the matrix (1.2). The condition (1.3) requires that the elements of $R_{1}$ and $R_{2}$ satisfy: $r_{11}+\widetilde{r}_{11}=1, r_{22}+\widetilde{r}_{22}=1, r_{12}+\widetilde{r}_{12}=0, r_{21}+\widetilde{r}_{21}=0, r_{11}^{2}+r_{12} r_{21}=r_{11}$,

$r_{12} r_{21}+r_{22}^{2}=r_{22}$,

$r_{12}\left(r_{11}+r_{22}-1\right)=0, r_{21}\left(r_{11}+r_{22}-1\right)=0$

Substituting (3.2) into the expressions (2.5) and (2.7) and using the fact that $r_{12}=-\widetilde{r}_{12}, r_{21}=-\widetilde{r}_{21}, \widetilde{r}_{11}=1-r_{11}, \widetilde{r}_{22}=1-r_{22}$ we get

$\left.\begin{array}{l}v_{11}(\alpha) / v_{21}(\alpha) \\ v_{12}(\alpha) / v_{22}(\alpha)\end{array}\right\}=\frac{-2 r_{12}\left(\beta_{1}-\beta_{2}\right)}{\left(\beta_{1}-\beta_{2}\right)\left(r_{11}-r_{22}\right) \mp\left\{\left(\beta_{1}-\beta_{2}\right)^{2}\left(r_{11}-r_{22}\right)^{2}+4\left(\beta_{1}-\beta_{2}\right)^{2} r_{21} r_{12}\right\}^{\frac{1}{2}}}$

$=-2 r_{12} /\left\{\left(r_{11}-r_{22}\right) \mp\left[\left(r_{11}-r_{22}\right)^{2}+4 r_{12} r_{21}\right]^{\frac{1}{2}}\right\}$

Now the conditions (3.4) require either (i) $r_{12}=r_{21}=0$ or (ii) $r_{11}+r_{22}=1$.

If (i) holds then $r_{12}=r_{21}=\widetilde{r}_{12}=\widetilde{r}_{21}=0$ and the original matrix is already in diagonal form (this corresponds to $\mathrm{v}_{11} / \mathrm{v}_{21}=\mathrm{v}_{12} / \mathrm{v}_{22}=0$ ).

If (ii) holds then from (3.3) we have $r_{21} r_{12}=-r_{22}^{2}+r_{22}=-\left(1-r_{11}\right)^{2}+\left(1-r_{11}\right)$ giving $r_{11}^{2}-r_{11}+r_{12} r_{21}=0$ so that $r_{11}=\left\{1+\sqrt{1-4 r_{12} r_{21}}\right\} / 2$. Also from (3.3) $r_{11}-r_{22}=2 r_{11}-1$. Thus $(2.15)$ becomes 


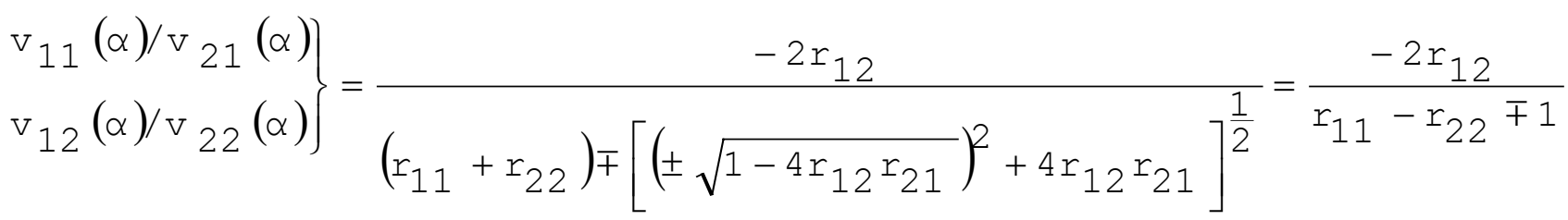

Clearly (3.6) satisfies the conditions (2.6) and (2.8) and therefore polynomial diagonalization can be carried out. The remaining matrices $G_{i}, i=3,4,5$ can also be shown to satisfy the conditions (2.6) and (2.8) and thus be Wiener-Hopf factorized by polynomial diagonalization.

In conclusion we suggest that given any $2 \times 2$ matrix that is required to be Wiener-Hopf factorized, the first test one should apply is that the elements satisfy (2.6) and (2.8). If these conditions are not satisfied, then consider the more sophisticated methods of factorization, see for example Heins[7], Daniele[8], Jones[9], Krapkov[10), Hurd[11], Rawlins and Williams[12], Rawlins[13], Williams[ 15]. A similar approach can be carried out for $3 \times 3$ matrices, however, although the cubic characteristic equation can be solved explicitly, the resulting formulae are complicated. It is probably better to apply the general principle of section 2 of this paper to $3 \times 3$ or indeed nxn matrices. This principle being that when one is solving the eigenvector equations one should choose the elements $v_{i j}(\alpha)$ of the transformation matrix such that they solve the eigenvalue equations and they are polynomials. 
[1] Lebre, A.B. Factorization in the Wiener Algebra of a class of $2 \times 2$ matrix functions. Integral Equations and Operator theory 12 (1989) pp408-423.

[2] Meister, E. and Speck, F-0, Wiener Hopf factorization of certain non-rational matrix functions in Mathematical physics. Preprint No.1156 Technische Hochschule Darmstadt (1988).

[3] Meister, E., and Speck, F-0, The explicit solution of the elastodynamical diffraction problems by symbol factorization. Z Anal. Ang. (to appear)

[4] Kupradze, V.D. (Ed) Three dimensional problems of the mathematical theory of Elasticity and Thermoelasticity. North-Holland Amsterdam 1979.

[5] Hurd, R.A. and Lüneburg, E, , Hurd, R.A. and Meister, E., Diffraction by an anisotropic impedance half plane. Can. Jour. Physc.63 (1985) pp1135-1140.

[6] Luneburg, E., Hurd, R.A. and Meister, E. Diffraction by a partially coated dielectric slab. Preprint No.1156. Technische Hochschule Darmstadt (1988).

[7] Heins, A.E. Systems of Wiener-Hopf integral equations and their application to boundary value problems in electromagnetic theory. Am. Symp. App. Math.2 (1948) pp76-81.

[8] Daniele, V.C. On the factorization of Wiener-Hopf matrices in problems solvable by Hurd's method. IEEE Trans. Ann. prop. AP-26 (1978) pp614-616.

[9] Jones, D.S. Factorisation of a Wiener-Hopf matrix. I.M.A. J. Appl. Math. 32 (1984) pp211-220.

[10]Krapkov, A.A. Certain cases of the elastic equilibrium of an infinite wedge with a non-symmetric notch at the vertex, subjected to concentrated forces. Pnkl. Mat. Mekch 35 (1971) pp625-637.

[ll]Hurd, R.A. The Wiener-Hopf-Hilbert method for diffraction problems. Can. Jour. Phys. 54 (1976) pp775-780.

[12]Rawlins A.D. and Williams W.E. Matrix Wiener-Hopf factorisation. Q. Jl. Mech.App.Math. 34 (1981) ppl-8.

[13] Rawlins A.D. Matrix Wiener-Hopf-Hilbert factorization. Siam J. App. Math. 46 (1986) ppl33-139.

[14]Williams, W.E. Recognition of some Readily Wiener-Hopf Factorizable Matrices. I.M.A. J. App. Math 32 (1984) pp367-378. 